\title{
Effects of Maternal Antenatal Performance on Newborn Care Practices and Birth Weight of Infants in the Bawku West District, Ghana
}

\author{
Article by Benjamin Aggrey \\ Master of International Health(MIH) and MPH, Texila American University, Ghana \\ Email: aggreyman4u@gmail.com
}

\begin{abstract}
BACKGROUND: Even though the Ghana Community-based Health Planning and Services (CHPS) initiative for scaling up service delivery is in place, the level of neonatal mortality remains high. OBJECTIVES: This study was designed to understand the current maternal antenatal performance and its effects on the birth weight of infants and selected new born care practices: Early initiation of breast-feeding; pre-lacteal feeding; Bathing of newborn after birth; Cord care; and Giving of water. METHODS: This community-based cross sectional survey combines both qualitative and quantitative data collection on a sample of 240 mothers and their babies; who were delivered in the community. RESULTS: A total of 81.7\% (196) of the women begun seeking Antenatal Care (ANC) during the first trimester of pregnancy and $91.7 \%$ (220) attended ANC sessions four or more times. Application of inappropriate substances to the umbilical cord was 79.2\% (190). Most women, 96.3\% (231), gave colostrum to their babies. Women who gave birth to normal weight babies were about two times more likely to have attended ANC as early as in the second trimester compared to women who did not give birth to normal weight babies (OR=1.69, CI: 0.740--3.867); $P=0.213$. Mothers who initiated ANC in the first trimester gave colostrum to their newborn infants compared to those in the second and third trimesters (OR=6.28, $\left.X^{2}=13.44, P=0.037\right)$. CONCLUSION: The study could not establish that early initiation of ANC in the first or second trimesters and maternal age at first delivery was independent predictors of the selected newborn care practices
\end{abstract}

Key Words: Maternal, Antenatal, New Born, Infants, Care

\subsection{Introduction}

According to Lawn, Cousens, and Zupan (2005) an estimated that each year 130 million infants are born worldwide out of which four million die in the first 28 days of life. Inappropriate care practices for the neonates are common in parts of Ghana resulting from rampant local traditions and culture. Bawku West District Annual Health Report (BWDAHR) (2014) indicate that in 2012, 2013, and 2014 newborn deaths that occurred in the District were 15, six (6), and five (5), respectively. In view of this trend that this research seeks to investigate maternal ANC seeking behaviors and how they influence their new born infant care practices.

\subsection{Objective}

To assess the ANC performance of women and its effect on the newborn care practices they adopt and the birth weight of their infants 0-11 months in the Bawku West District (BWD).

\subsection{Specific Objectives}

1. To establish relationship between degree of ANC performance and birth weight and selected newborn care practices 
South American Journal of Public Health

Special Edition May 2016

2. To determine the extent of selected newborn care practices respecting: Early initiation of breast-feeding; pre-lacteal feeding; Bathing of newborn after birth; Cord care; and Giving of water

\subsection{Methodology}

\subsection{Study Area}

The study took place in the Bawku West District in the Upper East Region (UER) of Ghana. The District has a total population of 98,630. Children 0-59 month old are 19,726; 011month, 3,945; 0-24 months, 7,890; and women in fertile age, 22,981. The District shares boundary with Burkina Faso in the north, Bawku Municipality in the East, and Nabdam District in the West. The District covers an area of approximately 90 square kilometers, constituting about $12 \%$ of the total area of the UER. The main occupation of the people is petty trading and subsistence crop and livestock farming. The District has one Hospital and two Medical Doctors. There are four (4) health Centers, 10 clinics and 18 other smaller health facilities (CHPS) spread across the seven sub-districts (BWDAHR, 2014).

The District has 134 communities that are largely rural and economically poor. The inhabitants of the communities adhere closely to local cultural practices in treating ailments an in caring for infants, despite regular health education provided by health workers. Malnutrition and illnesses among children under-five years are common. The BWDAHR (2014) indicates that teenage pregnancy is high in the District and in most cases; mothers and grandparents take care of the babies of these teenagers.

\subsection{Study Population}

The study population was all mothers, regardless of their age, who had 0-11 month's old infants of their own who had not attained their 12 months birthday. Records of their antenatal care consultations was examined from their ANC record booklets and their Children birth weights were checked and recorded from the Child Health Record Booklets; hence the target population was mother-infant pair. The respondents (mothers) were selected from all the seven sub-districts in the District to constitute the study sample.

3.2.1 Inclusion criteria. Respondents must be staying in the study area for more than a year; attended ANC, delivered and currently attending Postnatal Care (PNC) in any of the 31 health facilities in the study area regardless of ANC consultation status. Mothers must have their antenatal booklet and Child's Health Record Booklet at time of the interview.

\subsection{Study Design and Duration}

The study was carried out from $13^{\text {th }}$ October, 2014 to $25^{\text {th }}$ January, 2015. This communitybased cross sectional survey combined both qualitative and quantitative data collection methods through interviews with a structured schedule and an interview guide for the focus group interview. The study also has diagnostic component as it sought to determine the frequency of maternal antenatal attendance and their association with infants' feeding frequency.

\subsection{Sampling Approach and Process}

3.4.1 Sampling process. District Annual Health Report shows that women who had children aged five (5) years who attended postnatal clinic in October, 2014 were 5,505 in the entire District; 3,945 of these children were between 0-11months old (Bawku West District Annual Health Report, 2014)

A sample size of 240 mother-infant pairs was obtained from a universe of 3,945 in 366 households in 28 randomly selected communities. The women must have attended both ANC and postnatal services in any of the 31 health facilities in the District at the time of the interview. Each of the seven (7) sub-districts constituted a cluster that has an average of four (4) health facilities, in different communities. The communities were purposively selected based on proximity to health facilities. The sample size was drawn from seven clusters of 
communities. Random sampling technique was employed to select at least two (2) communities from each cluster to ensure district-wide representation of respondents. Each of the selected communities constituted a sampling frame. Simple random sampling method was employed to select houses from each frame. In each of the compound house entered, interviewers interviewed every mother in a household who had a child within the specified age group. Total respondents from all the frames represented the total sample size of the survey.

\subsection{Sample Size Estimation}

A Sample size of 240 was calculated using the 2011 version of Essential Nutrition Action (ENA) software. The prevalence of severe and moderate underweight among children 0-11 months was $10.7 \%$ and a percentage of children less than two years were $8.0 \%$, using the district population. The desired precision of 5.0\%; design effect of 1.5; household size of five (5); percentage of non-response households three (3); and the total households of 366 were used in the calculation of the sample size.

\subsection{Data Collection Tools}

A Structured schedule and an interview guide were developed for both Quantitative and Qualitative data. The tools were developed following extensive literature review on maternal antenatal service utilization and home-based newborn care practices. The Schedule had components on respondents and household characteristics, ANC, newborn care practices, nutrition, and postnatal care for mother and baby.

\subsection{Pretesting and Data Collection}

The schedule was field-tested and necessary changes made before data collection was initiated by three (3) teams of two (2) interviewers each and a supervisor. All data collectors were knowledgeable in Health Surveys. Before start of the data collection exercise, one day training was organized for the data collectors and supervisors to enhance their skills on the research protocols and study objectives.

\subsection{Data Analysis}

Checks for range and internal consistency were performed regularly. Queries were generated from consistency checks after data entry and from validation checks on the databases. All the schedules were reviewed and coded in the case of open-ended responses. Data was analyzed using SPSS software. Univariate analyses were expressed as frequencies, percentages, and mean. Bivariate analysis was done to determine the association between antenatal care performance and birth weight and the newborn care practiced. Chi-square $\left(\mathrm{X}^{2)}\right.$ test was used to find out significant differences between variables. Statistical associations between ANC performance and newborn care practices and birth weights were measured using both crude estimates of associations and associations adjusted for the confounding effect of the included confounding variables (Logistic regression model). The results were then presented as odds ratios with $95 \%$ CI. For all tests, p-value of $<0.05$ was judged significant. For analyzing qualitative data, available responses were freely listed, qualified and semi-quantified. Qualifiers for summarizing information gathered after every Group Discussions were semi-quantified based on the investigators judgment (Dey, 1993).

\subsection{Ethical Considerations}

The research started only after appropriate ethical clearance had been sought from the Regional Health Directorate, UER, where this research was carried out. Strict confidentiality was maintained during processing and analysis of the data. Respondents were informed about the rationale of the study before interviews begun. Female interpreters were recruited to interpret more sensitive women-related questions. Respondents were assured of the confidentiality of their responses. Respondents who did not consent to answer questions were allowed to opt out of the study. 
South American Journal of Public Health

Special Edition May 2016

\subsection{Limitations of Study and Potential Bias}

Since the study is a cross-sectional survey, no causality could be determined. The results should only be viewed in the context of BWD, not the entire UER, since there might be cultural difference in other parts of the region. There might be a recall bias on the part of some respondents whose infants might be close to 11 months old. Measurements of certain extraneous variables could not be made due to limited time for the study.

\subsection{Observations and Findings}

\subsection{Maternal Demography}

A total of 240 mothers were recruited in the study, giving response rate of $100 \%$. The mean age of the respondent (mothers) at the time of the interview was 27 years with minimum age being 18 years and maximum age, 42 years. Table one (1) indicates that majority of women were married (98.3\%). Out of these, 61.3\% (147) had their first delivery within 15 to 19 years of age.

Table 1: Socio-Demographic Characteristics of Mothers

\begin{tabular}{lll}
\hline Variable types (N=240) & Frequency (n) & Percentage (\%) \\
\hline Maternal current age & 19 & 8.0 \\
15-19 years & 163 & 67.9 \\
20-30 years & 58 & 21.2 \\
31-45 years & & \\
& & \\
Maternal age at first delivery & 147 & 61.3 \\
15-19 years & 91 & 37.9 \\
20-30 years & 2 & 0.8 \\
31-45 years & & \\
& & \\
Marital status & 236 & 98.3 \\
Married & 1 & 0.4 \\
Not Married & & \\
Parity & 65 & 27.1 \\
One Child & 175 & 73.0 \\
Two or More & & \\
\end{tabular}

Out of the 240 mothers, $81.7 \%$ (196) had some income generating work to do while $15.8 \%$ (38) did not have any. The reasons given for not working was either being a student or being a house wife and others unable to do any other income earning work at all. The main occupation of the respondents' were subsistence farming (38.3\%) followed by petty trading (Figure 1). Those who delivered at the age of 20 to 30 years were 37.9\% (91). Those with no formal education were $68.8 \%$ (165). Those whose formal education terminated at primary six were $15 \%$ (36) and only $2.1 \%$ (5) had tertiary education (Figure 2).

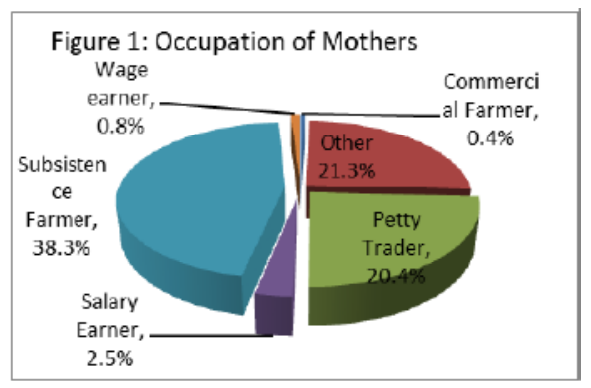




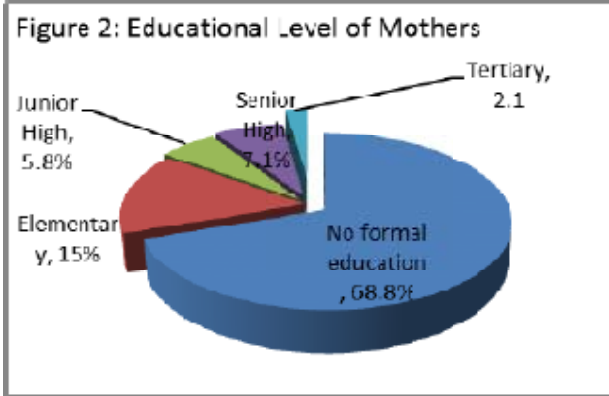

\subsection{Infant Demography}

The mean age of the infants was 4.96 months and Standard Deviation (SD) of 2.9 months. Birth weight of the infant range from $1.8 \mathrm{~kg}$ to $4.0 \mathrm{~kg}$ and the mean weight was $2.9 \mathrm{~kg}$. Males were $51.7 \%$ (124) and females were $48.3 \%$ (116). The mean height of the infants was $70.1 \mathrm{~cm}$ (SD $4.072 \mathrm{~cm}$ ). Table one (1) shows birth weights below $2.5 \mathrm{~kg}$ were $7.6 \%$ (18) while $92.4 \%$ (220) weighed greater than or equal to $2.5 \mathrm{~kg}$.

\subsection{Maternal ANC Performance}

Table two shows $54.6 \%$ of the respondents sought ANC through their personal initiative while others were encouraged by relatives (16.3\%). The study also found that $81.7 \%$ (196) of the women begun seeking ANC during the first trimester of pregnancy. Majority (91.7\%) of the women attended ANC sessions four or more times and only two persons (1.3\%) attended ANC only one time before delivery (Figure 3).

Table 2: Maternal ANC Performance

\begin{tabular}{lll}
\hline ANC Performance (N=240) & $\begin{array}{l}\text { Frequency } \\
\text { (n) }\end{array}$ & Percentage (\%) \\
\hline & & \\
Source of information about & & \\
ANC & 8 & 3.3 \\
From a friend & 35 & 14.6 \\
Health Professional & 17 & 7.1 \\
Other (specify) & 131 & 54.6 \\
Personal initiative & 2 & 0.8 \\
Radio & 39 & 16.3 \\
Told by relative & 5 & 2.1 \\
When visited clinic before & & \\
pregnancy & & \\
ANC initiation & 196 & 81.7 \\
First trimester & 38 & 16.0 \\
Second trimester & 4 & 1.7 \\
Third trimester &
\end{tabular}

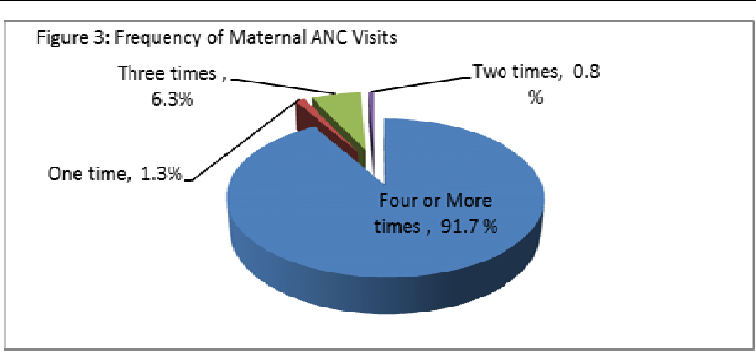


South American Journal of Public Health

Special Edition May 2016

\subsection{New Born Care Practices}

Most of the women (90.8\%) delivered at various health facilities in the District. More than half (60.4\%) of the women were not visited at home by health staff (Table 3).

4.4.1 Giving of water to infant. Out of the 240 interviewed, $45.9 \%$ (110) of mothers gave water to their infants before they attained six months of age while $18.8 \%$ and $27.1 \%$ gave water during the first month and before six months, respectively. Only 14.6\% (35) gave water to infants after six months of age (Table 3). Inappropriate giving of water to neonates was 85.4\% (205), (Figure 5).

4.4.2 Bathing of newborn after birth. The results revealed that after delivery, mothers or mothers-in-law of the newly delivered women bathed the newborn babies, $68.3 \%$ (164). Only $17.5 \%$ (42) of the mothers bathed their own babies. The remaining $14.2 \%$ (34) of the babies were bathed, either by rival (husband's other wife), sister, sister in-law, friends or grandmother of the newly delivered woman. Most of the respondents recalled bathing of their newborns was done any time after six hours (75.8\%); 19.2\% (46) bathed newborns after 24hours, Table 3. In their view the right time to bathe newborn infants should be within six hours, 36\% (88); immediately after birth, 33\% (80); between six and 24hours, 3.3\% (8); up to 24hours 4.2\% (10); days after delivery 2.9\% (7).

4.4.3 Safe cord care. The results indicate that $62.5 \%$ (148) of the mothers applied substances while $37.5 \%$ (92) applied nothing at all. Others admitted using variety of substances to treat the cord (22.1\%). Other substances applied to the cord were either applied alone or mixed with Shea Butter. These include "tying cord with a black thread"; certain herbs; fresh tomatoes and salt; Pepsodent (toothpaste) and herbs; charcoal and shea butter; and herbs ("Gbampru") and Shea Butter. Other substances applied include: "Bankot", "Gumpo", cow dung, chalk, burnt maize, and Magi. In all, majority (79.2\%) of the women applied inappropriate substances to the umbilical cord of the neonates (Figure 4).

4.4.4 Breastfeeding. Most of the women, 96.3\% (231), gave colostrum to their babies after delivery. Women who breastfed their newborns within 30 minutes after delivery were $57.1 \%$ (137) and those who put their newborns to breast within one hour were 32.5\% (78), Table 3. As indicated in Figure five, $79.6 \%$ of the women breastfed their newborns appropriately (at least 7 times a day) while appropriate breastfeeding initiation was 89.6\% (215).

Table 3: Delivery, Newborn and Postnatal Care practices

\begin{tabular}{lll}
\hline Newborn Care Practices (N=240) & Frequency (n) & Percentage (\%) \\
\hline Place of delivery & 218 & 90.8 \\
Health facility & 15 & 6.3 \\
Home & 5 & 2.1 \\
On the way to facility & 145 & 60.4 \\
& & \\
Visited at home by any nurse? & 93 & 38.8 \\
No & & \\
Yes & 23 & 9.6 \\
Initiation of breast-feeding after delivery & \\
After one or more days & 137 & 57.1 \\
Within 30 minutes & 78 & 32.5 \\
Within one hour & 40 & 16.7 \\
I don't know & & \\
& & \\
Giving of water to infants & 159 & 66.3 \\
No & 79 & \\
Yes & &
\end{tabular}

Age at which water was given to infants 
South American Journal of Public Health

Special Edition May 2016

First month

Before six months

After six months

Other responses

Bathing of infants after delivery

After 24 hours

At least six (6) hours of birth

Substance application to cord

No
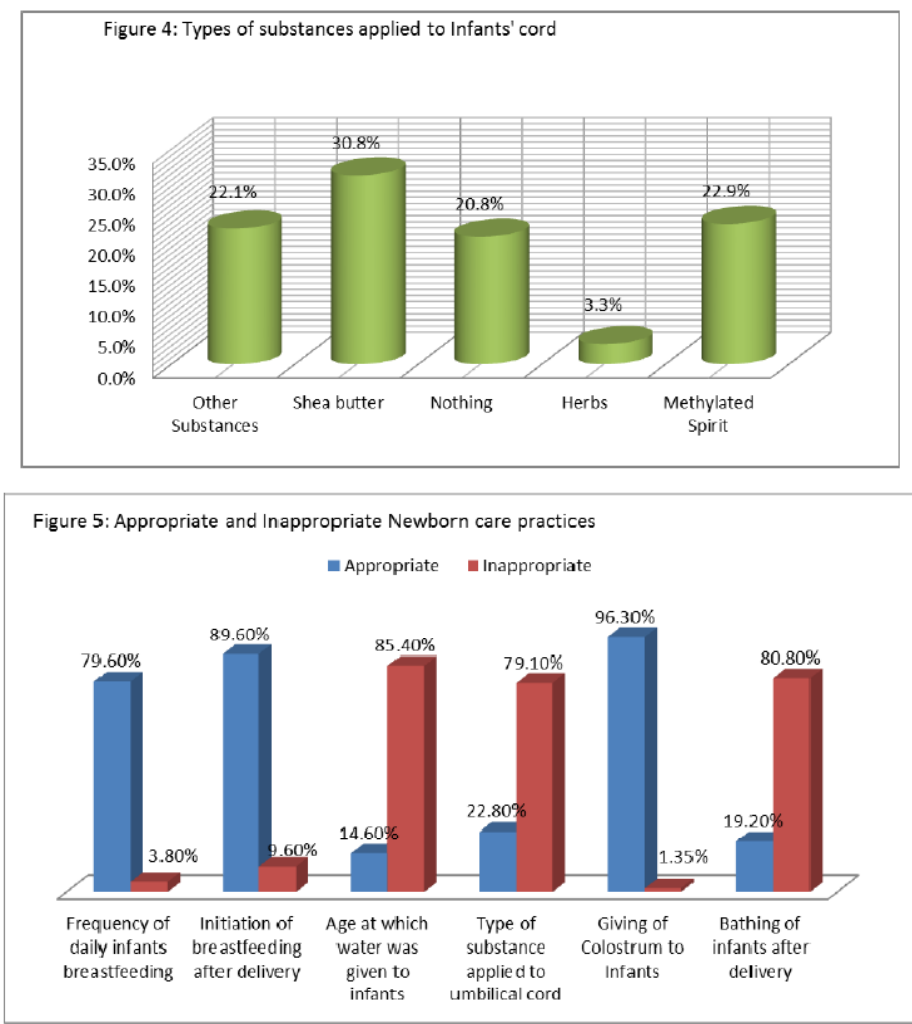

Table 4: Predictors of Normal Birth Weight of Newborn Infants

\begin{tabular}{lllllll}
\hline & & Wald & Sig. & $\operatorname{Exp}(\mathrm{B})$ & \multicolumn{2}{l}{ 95\% CI for Exp(B) } \\
\cline { 6 - 7 } & & & & & Lower Bound & Upper Bound \\
\hline 2nd Trimester & Intercept & 6.176 & 0.013 & & & \\
& Weight at Birth & 1.553 & 0.213 & 1.692 & 0.740 & 3.867 \\
3rd Trimester & Intercept & 0.074 & 0.785 & & & \\
& Weight at Birth & 0.750 & 0.387 & 0.355 & 0.034 & 3.699 \\
\hline
\end{tabular}

Table 5: Predictors of Mothers Giving Colostrum to Newborn Infants

\begin{tabular}{|c|c|c|c|c|c|c|}
\hline & & \multirow[t]{2}{*}{ Wald } & \multirow[t]{2}{*}{ Sig. } & \multirow[t]{2}{*}{$\operatorname{Exp}(B)$} & \multicolumn{2}{|c|}{ 95\% CI for $\operatorname{Exp}(B)$} \\
\hline & & & & & $\begin{array}{l}\text { Lower } \\
\text { Bound }\end{array}$ & $\begin{array}{l}\text { Upper } \\
\text { Bound }\end{array}$ \\
\hline \multirow{2}{*}{$\begin{array}{l}\text { 2nd } \\
\text { Trimester }\end{array}$} & Intercept & 4.250 & 0.039 & & & \\
\hline & $\begin{array}{l}\text { Colostrum given to } \\
\text { Infants }\end{array}$ & 0.613 & 0.434 & 2.635 & 0.233 & 29.814 \\
\hline 3rd & Intercept & 398.080 & 0.000 & & & \\
\hline
\end{tabular}


South American Journal of Public Health

Special Edition May 2016

Trimester
Colostrum given to
8.616
8.616
8.616

Infants

Table 6: Predictors of Application of Substances to the Umbilical Cord of newborns

\begin{tabular}{|c|c|c|c|c|c|c|}
\hline & & \multirow[t]{2}{*}{ Wald } & \multirow[t]{2}{*}{ Sig. } & \multirow[t]{2}{*}{$\operatorname{Exp}(B)$} & \multicolumn{2}{|c|}{ 95\% CI for $\operatorname{Exp}(B)$} \\
\hline & & & & & $\begin{array}{l}\text { Lower } \\
\text { Bound }\end{array}$ & $\begin{array}{l}\text { Upper } \\
\text { Bound }\end{array}$ \\
\hline \multirow{2}{*}{$\begin{array}{l}\text { 2nd } \\
\text { Trimester }\end{array}$} & Intercept & 2.268 & 0.132 & & & \\
\hline & $\begin{array}{l}\text { Substance Application } \\
\text { to Cord }\end{array}$ & 2.518 & 0.113 & 0.533 & 0.245 & 1.159 \\
\hline \multirow{2}{*}{$\begin{array}{l}\text { 3rd } \\
\text { Trimester }\end{array}$} & Intercept & 7.877 & 0.005 & & & \\
\hline & $\begin{array}{l}\text { Substance Application } \\
\text { to Cord }\end{array}$ & 0.158 & 0.691 & 1.494 & 0.206 & 10.824 \\
\hline
\end{tabular}

Table four (4) shows women who attended ANC in the second trimester were about two times likely to give birth to normal weight babies (OR=1.69, CI: 0.740--3.867); $\mathrm{P}=0.213$. Women who initiated ANC in the second trimester were two times less likely to give colostrum to their newborn infants compared to those who initiated ANC in the third trimester ( $\mathrm{OR}=2.63$, CI: 0.233--29.814); $\mathrm{P}=0.613$. As indicated in the Table 6, there is no association between second trimester initiation of ANC and application of substances to the umbilical cord of infants (OR=0.53, CI: 0.245--1.159); $\mathrm{P}=0.113$.

Table seven (7) shows that mothers who initiated ANC in the first trimester gave colostrum to their newborn infants compared to those in the second and third trimesters $(\mathrm{OR}=6.28$, $\left.X^{2}=13.44, P=0.037\right)$. No significant association was found between Maternal Age at First Delivery and Giving of Colostrum to Newborn Infants (OR=3.39, $X^{2}=2.31, P=0.679$. Table eight (8) shows maternal ANC initiation had no significant relationship with application of substance to the umbilical cord of the newborns $\left(\mathrm{OR}=3.17, X^{2}=2.75, P=0.839\right)$. The test also showed no significant association between maternal age at first delivery and application of substance to the umbilical cord of the newborns $\left(\mathrm{OR}=4.54, X^{2}=3.87, P=0.424\right)$. Maternal age at delivery and infants' birth weight did not show any significant association $\left(\mathrm{OR}=13.04, X^{2}=\right.$ 12.23, $\mathrm{P}=$ 0. 933), (Table 9).

Table 7: Relationship between giving of Colostrum to Infants and Maternal ANC Initiation and Age at first delivery

\begin{tabular}{|c|c|c|c|c|}
\hline \multirow[t]{2}{*}{$\begin{array}{l}\text { Trimester of ANC } \\
\text { initiation }\end{array}$} & \multicolumn{2}{|c|}{$\begin{array}{l}\text { Giving of Colostrum to } \\
\text { Infants(N=240) }\end{array}$} & \multirow[b]{2}{*}{ Total } & \\
\hline & No & Yes & & \\
\hline First trimester & $2(0.8 \%)$ & 199 (82.9\%) & 207 (85.0\%) & \\
\hline Second trimester & $1(0.4 \%)$ & $36(15.0 \%)$ & 37 (15.4\%) & $O R=6.28$ \\
\hline Third trimester & $0(0.0 \%)$ & $2(0.8 \%)$ & $2(0.8 \%)$ & $X^{2}=13.44$ \\
\hline Count (\% of Total) & $3(1.3 \%)$ & 237 (98.8\%) & $24(100.0 \%)$ & $P=0.037$ \\
\hline \multicolumn{5}{|c|}{ Maternal Age at First } \\
\hline $15-19$ years & $3(1.3 \%)$ & $144(60.1 \%)$ & 150 (64.6\%) & \\
\hline 20-30 years & $0(0.0 \%)$ & 91 (38.0\%) & 94 (39.2\%) & $O R=3.39$ \\
\hline 31-45 years & $0(0.0 \%)$ & $2(0.8 \%)$ & $2(0.8 \%)$ & $X^{2}=2.31$ \\
\hline Count (\% of Total) & $3(1.3 \%)$ & 237 (98.8\%) & $24(100.0 \%)$ & $P=0.679$ \\
\hline
\end{tabular}

Table 8: Relationship between substance applications to cord and Maternal ANC Initiation and Age at first delivery

Trimester of ANC $\quad$ Application of Substances $\quad$ Total




\begin{tabular}{lllll}
\hline \multirow{2}{*}{ initiation } & \multicolumn{2}{l}{ to Cord (N=240) } & \\
\cline { 2 - 3 } & No & \multicolumn{2}{l}{ Yes } & \\
\hline First trimester & $79(32.9 \%)$ & $120(50.8 \%)$ & $201(84.6 \%)$ & \\
Second trimester & $10(4.2 \%)$ & $27(11.3 \%)$ & $37(15.4 \%)$ & $O R=3.17$ \\
Third trimester & $1(0.4 \%)$ & $1(0.4 \%)$ & $2(0.8 \%)$ & $X^{2}=2.75$ \\
$\begin{array}{l}\text { Count (\% of Total) } \\
\text { Maternal Age at First }\end{array}$ & $90(37.5 \%)$ & $148(62.5 \%)$ & $24(100.0 \%)$ & $P=0.839$ \\
Delivery & & & & \\
15-19 years & $49(20.4 \%)$ & $98(40.8 \%)$ & $149(62.1 \%)$ & \\
20-30 years & $40(16.7 \%)$ & $51(21.3 \%)$ & $91(37.9 \%)$ & $O R=4.54$ \\
$31-45$ years & $1(0.4 \%)$ & $1(0.4 \%)$ & $2(0.8 \%)$ & $X^{2}=3.87$ \\
Count (\% of Total) & $92(37.5 \%)$ & $148(62.5 \%)$ & $24(100.0 \%)$ & $P=0.424$ \\
\hline
\end{tabular}

Table 9: Relationship between Maternal Age, Frequency of ANC Visits and Birth Weight (kg) of Infants

\begin{tabular}{llllllllll}
\hline Maternal & \multicolumn{1}{l}{ Infant Weight at Birth (N=240) } & & Total \\
\cline { 2 - 8 } Age at First & $(1.5-$ & $(2.0-$ & $(2.5-$ & $(3.0-$ & $(3.0-$ & $(3.4-$ & $(3.5-$ & $(4.0-$ & \\
Delivery & $1.9)$ & $2.4)$ & $2.9)$ & $3.3)$ & $3.4)$ & $3.9)$ & $3.9)$ & $4.4)$ & \\
\hline 15-19 years & 2 & 9 & 68 & 0 & 55 & 2 & 11 & 0 & 147 \\
& $0.8 \%$ & $3.8 \%$ & $28.3 \%$ & $0.0 \%$ & $22.9 \%$ & $0.8 \%$ & $4.6 \%$ & $0.0 \%$ & $61.3 \%$ \\
\hline $20-30$ years & 1 & 8 & 35 & 1 & 29 & 3 & 13 & 1 & 91 \\
& $0.4 \%$ & $3.3 \%$ & $14.6 \%$ & $0.4 \%$ & $12.1 \%$ & $1.3 \%$ & $5.4 \%$ & $0.4 \%$ & $37.9 \%$ \\
\hline $31-45$ years & 0 & 0 & 0 & 0 & 1 & 0 & 1 & 0 & 2 \\
& $0.0 \%$ & $0.0 \%$ & $0.0 \%$ & $0.0 \%$ & $0.4 \%$ & $0.0 \%$ & $0.4 \%$ & $0.0 \%$ & $0.8 \%$ \\
\hline Count & 3 & 17 & 103 & 1 & 85 & 5 & 25 & 1 & 240 \\
$\%$ of Total & $1.3 \%$ & $7.1 \%$ & $42.9 \%$ & $0.4 \%$ & $35.4 \%$ & $2.1 \%$ & $10.4 \%$ & $0.4 \%$ & $100.0 \%$ \\
\hline \multicolumn{2}{l}{ OR=13.04, $X^{2}=12.23, P=0.933 ;$} \\
\hline
\end{tabular}

\subsection{Group Interview}

Interviews were held with two focus groups to seek their views about the type of care offered to newborn babies in their communities. One group was women who have had delivery experience but are no longer delivering children; they were between the ages of 30 and 60 years (referred to as Older Women). The second group was women who were then nursing their own newborn infants (referred to as nursing mothers). Each group consisted of ten participants.

4.5.1 Perception on ANC. According to the two groups (90\%), ANC is the process of visiting a health facility when a woman is pregnant. The women unanimously agreed that a pregnant woman should attend ANC clinic at least eight times before delivery and the nursing mothers said they were educated to visit ANC as soon as pregnancy is detected and on monthly basis, but near the end of gestation, they were to attend ANC twice per month and finally on weekly basis until they deliver.

4.5.2 Breast-feeding. According to the older women, babies should be breastfed two hours after delivery when delivery occurred at home; some also breastfeed three to four hours after delivery, from their common experience. At the health facilities, they said, breastfeeding should be 30 minutes to one hour after delivery. They also said that newborn babies can breastfeed at least 10 or more times per day. The nursing mothers, however, said after delivery, if mother and baby are in good health after delivery, they immediately breast-feed the child, but it takes so many hours before breastfeeding can be established due to condition of mother and child. They were of the view that mothers can stop breastfeeding at their own time; when child is two or three years (80\%).

Some of the older women (70\%) felt there is no need adding anything to breast milk until infants attain sixth months because the breast milk contains the water baby will needs up to $6^{\text {th }}$ month. Some also felt sometimes the "breast milk do not flow well and others taste bitter 
South American Journal of Public Health

Special Edition May 2016

so there is the need to add water and "weanimix" porridge." Some of the women (30\% of older women) felt that breast-feeding can stop depending on the health status of the child, while the rest felt that it can continue until two or three years.

4.5.3 Cord care. Some of the older women (40\%) said during their time, there were one or two people who went to homes to bathe the newborns but now it is the mother's in-law or any other person who knows how to bathe babies. They said, in the olden days, they used local herbs (Gbampru) on the cord to help the cord fall sooner and heal; others also used Shea Butter to prevent dryness of cord but now, mothers use baby towel to clean the cord and leave it to dry. They women felt that some people in the community might be using local treatment of the cord because.

All the women groups unanimously declared that the main challenges they see women face in newborn care is that some children cry a lot and others fall sick frequently, hence, not allowing mothers do much work to help themselves. "Some newborns also suck a lot of breast milk; most women get waist pains out of breastfeeding; sometimes women are unable to sleep well at night due to the continual suckling and crying of the babies." The women said that it is during their first delivery that they face most of these challenges but the subsequent ones are more manageable.

The nursing mothers admitted that there were many challenges with breast-feeding, especially in the first month of delivery. These included difficulty in lifting the newborn in the first few days; others also cited exposure of the newborn to outside world (out of room) is usually too early because of poor economic state of the family; they were sometimes forced to send the newborn outdoors to engage in income generating activities and household chores.

\subsection{Discussion}

This study tried to retrospectively examine the ANC performance of postnatal women in the BWD and the effect it has on their new born care and birth weight of their newborns. The women interviewed, in general, were very cooperative in their responses.

Among the findings was that majority of the mothers in the study did not have any formal education (68.8\%); similar to a previous studies carried out in northern Ghana which found similar higher rate of no formal education among mothers (88.6\%) of infants (MICS, 2011). The effects of maternal low or no formal education on child care has been established by other studies such as Mangwi, Kasasa, Criel, Garimoi, and Kolsteren (2014) which reported that maternal higher education and complete cord care were positively associated.

The fact that most of the women (90.8\%) delivered at various health facilities is an indication that they received assistance from trained midwives and other health professionals. Women are encouraged to seek ANC several times on monthly basis to enable the nurses identify any emerging danger signs and offer appropriate and timely interventions to elude any negative impact on the newborn and the mother. This study found that most (91.7\%) of the women attended ANC four or more times before they delivered. This performance is higher than the national (Ghana) performance of $86.6 \%$ and the Upper East Regional performance of $88.8 \%$, according to the MICS (2011) report. Although this study could not establish any significant association between number of ANC attendance by pregnant women and its effects on the newborn infants, other studies have shown that it is protective for mothers and their newborns if a pregnant woman seeks adequate ANC. This was proven by a study conducted in Uganda which established significant association between four or more ANC consultations and complete cord care (MICS, 2011; Mangwi et al, 2014; and Abhulimhen-Iyoha, Ibadin, 2012). Neupane and Nwaru (2014) affirm that children of mothers with no prenatal care were at increased risk of neonatal death $(\mathrm{OR}=2.03,95 \% \mathrm{CI}=1.28$ 3.23). Another study conducted in Karachi, Pakistan, found that $70 \%$ of the women interviewed received ANC by skilled providers and 54.4\% sought four or more times before they delivered. When women deliver at home, it endangers their own lives because when complications such as postpartum hemorrhage, obstructed labor, eclampsia, etc. set in, both the woman and the baby may lose their lives. When women deliver in the health facility, they 
are able to receive facility-based, skilled, postnatal care for at least 24 hours after birth and subsequently, home visits on the third day (48-72 hours); and six weeks after birth, in accordance with the WHO recommendation for postnatal care and current Home-based Newborn Care program being implemented in some districts in Ghana, including BWD, by GHS. This is in contrast with home delivery where it might be very difficult for health personnel to trace the newly delivered mother and her baby to offer the necessary assistance like administering BCG, if the woman did not willingly report at the health facility. This study found that more than half $(60.4 \%)$ of the women were not visited at home by health staff (Community health Nurses) who were supposed to do same.

Regarding cord care, this study found that few (22.80\%) mothers of the infants practiced good cord care, comparable to a study conducted in Uganda and Bangladesh which found, respectively, that $42.8 \%$ and $23.7 \%$ of the women interviewed were considered to have practiced good cord care. They showed that application of substances to the cord was not significantly influenced by early or late initiation of ANC by pregnant women (Mangwi et al, 2014 and Gary et al, 2005). The Ghana Under-Five Child Health Policy (2007-2015) stipulates that nothing, apart from clean water, should be used to clean the cord of new born infants. Contrary to this policy, the study found that poor cord care was practiced by majority of the women (75.8\%); this was evident in the type of substances used to treat the cord. They included ligature of the cord with black thread and applying certain herbs; Tomatoes and salt; tooth paste (Pepsodent) plus herbs; magi, herbs ('Gbampru’ and Shea Butter), "Bankot", Seit, "Gumpo", Cow Dung, Charcoal and Shea Butter, Chalk, Burnt Maize. These, they believe, could heal and speed up the falling of the cords. Other researchers found similar practices elsewhere (Mangwi et al, 2014; Marah, 2011; and Mahama and Mariam, 2014). The likelihood that most of the aforementioned locally prepared medicines could introduce infections to the cord is very high due to the unhygienic environment that these medicines are usually prepared, and can eventually cause sepsis in the newborn. This could be a contributing factor to the prevailing newborn deaths in the study district (BWDAHR, 2014). It is noteworthy then that most of these practices mostly occurred in the homes of these women after discharged from the health facility, situation similar to what Barnett et al (2006) found in a study conducted in Bangladesh.

Safe cord care was practiced by $20.8 \%$ of the women, who said nothing was applied to the infant's cords before they dropped. If these and other future mothers in the communities are not given regular health education, at the antenatal and postnatal clinics, on the need for families to avoid application of potentially harmful substances to the umbilical cords of newborns, there is likelihood that influential family members, especially mothers-in-law, will pressurize the newly-delivered women to slip into adopting these unhealthy practices. Even though the study did not find out why nothing was applied to the cord by those who did not do so, it could be as a result of their adherence to the regular health education given by health staff during ANC sessions; it could also be the result of different perception which might need further investigation in the near future to ascertain the reality.

The fact that most of the mothers were aware of the importance of seeking ANC services is an indication that the GHS program of regular education offered to women during all antenatal and postnatal clinics might be having positive influence on the women in the BWD, thus most of them knew that attending ANC more than four times before delivery was good for both the pregnant woman and the unborn baby. This is in line with the Ghana Health Service policy that all pregnant women attend ANC services at least four times before they give birth (Ghana Under-Five Child Health Policy, 2007-2015).

The results of this study further showed that mothers who initiated ANC in the first trimester of pregnancy gave colostrum to their newborn infants compared to those in the second and third trimesters $\left(X^{2}=13.44, P=0.037\right)$. This can be attributed to the fact that the regular health education rendered to pregnant women during ANC is having positive impact on them. Therefore, since the educational topics vary during these sessions, those women who 
South American Journal of Public Health

Special Edition May 2016

attend antenatal clinic early and frequently are likely to be reminded more often about the importance of breastfeeding.

Concerning infants' bathing after delivery, the fact that only $19.2 \%$ were bathed after 24hours suggests that more infants might be exposed to early bathing and the danger of hypothermia which can easily cause newborn deaths. Similarly, a study conducted in Bangladesh found that $65 \%$ of the infants were bathed before 24 hours' time limit, and $44 \%$ were bathed immediately after birth (Barnett et al, (2006). Early bathing of infants appears to be a common practice by various cultures in Ghana, according to the findings of this study and other studies conducted in Northern Ghana (Mahama and Mariam, 2014). where varied cultural practices, coupled with notion that the newborn might be stained with blood during the delivery process. It can also be as a result of the notion that newborns can be dirty due to the fact that our environment is naturally warmer and dustier. According studies conducted in Uganda and elsewhere, early bathing significantly lowered neonatal body temperatures even when bathed with warm water and later applied skin-to-skin warming for the baby (Mangwi et al, 2006; Bergstrom, Byaruhanga, Okong, 2005; Gilani and Kayani, 2014).

This study found that $89.6 \%$ of the women initiated breastfeeding timely (within one hour of birth), slightly higher than another study done in Ghana in which timely initiation of breastfeeding rate was less than $80 \%$ (Mahama and Mariam, 2014). There are a lot of beneficial reasons a newly-delivered woman should introduce the newborn to the breast milk as early as within one hour of birth. Among these reasons are that the infant will begin feeding on a perfect mix of nutrients laden with immune factors (colostrum). The early initiation of breast milk serves as the beginning of strong bonding between mother and the baby. The first breast milk also contains colostrum, which is highly nutritious and has antibodies that protect the newborn from diseases (Mahama and Mariam, 2014). The early suckling of the breast stimulates breast milk production and eases the release of Oxytocin, which in turn helps the contraction of the uterus and reduces postpartum blood loss.

This study found that most of the women could not observe the exclusive breastfeeding policy that nothing else, except medically indicated, should be given to the newborns; neither water, infant feed, nor other substances. However, $45.9 \%$ of the women gave water to their infants before the infants attained six months of age. This noncompliance attitude could be as a result of some family members still feeling that because Northern Ghana is typically sunny and warmer, water is then needed as a complement to the breast milk to quench infants' thirst. This practice might also be perpetrated by other loved ones who bathed the babies in support of the newly-delivered women, especially mothers-in-law (68.3\%), who might sometimes give the water under cover, especially if the parents of the baby might not agree with them. Women who breastfed newborns appropriately were relatively high in this study (79.6\%). This is comparable to another study (Hall and Carl, 2002) which found exclusive breastfeeding (87.6\%) as one of the beneficial newborn care practices. However, further cross-sectional study needs to be conducted to find out why in the focus group interview with the non-delivering women, they said that babies should be breastfed after two hours when delivery occurs at home but if it occurs in a health facility, breastfeeding should begin 30 minutes to one hour after delivery; there might be an underlining local practice yet to be known.

\subsection{Conclusions}

The study revealed that inappropriate new born care practices by mothers and family members is prevalent in the BWD, especially among the rural inhabitants who usually adopt certain cultural practices on newborn care. In Contrast with the WHO recommendations on newborn care and findings by other researchers, this study could not establish that early initiation of ANC in the first or second trimesters were independent predictors of the selected newborn care practices, neither was the study able to establish that the independent variables are predictors of infants' birth weight. 


\subsection{Recommendations}

Ghana Health Service staff in the District ought to deepen their efforts in behavior change communication and counseling of mothers and caregivers on CIYCF practices, in collaboration with local stakeholders. Provision should be made for motor bikes for the Community Health Nurses who are duty-bound for home visits to pay adequate visits to the homes of the pregnant women and the newly delivered mothers and their newborn infants.

\section{References}

[1.] Abhulimhen-Iyoha BI, Ibadin MO (2012). Determinants of cord care practices among mothers in Benin City, Edo State, Nigeria. Niger J Clin Pract. 2012 Apr-Jun;15(2):210-3. doi: 10.4103/11193077.97320. PubMed PMID: 22718175.

[2.] Barnett S, Azad K, Barua S, Mridha M, Abrar M, Rego A, Khan A, Flatman D, Costello A. (2006) Maternal and newborn-care practices during pregnancy, childbirth, and the postnatal period: a comparison in three rural districts in Bangladesh. J Health Popul Nutr. 2006 Dec;24(4):394-402. PubMed PMID: 17591336; PubMed Central PMCID: PMC3001143.

[3.] Bawku West District Annual Health Report (2014). Annual Health Report of the Bawku West District Health Directorate.

[4.] Bergstrom A, Byaruhanga R, Okong P. (2005). The impact of newborn bathing on the prevalence of neonatal hypothermia in Uganda: a randomized, controlled trial. Acta Paediatr 2005, 94(10):14621467.

[5.] Dey I. (1993). Qualitative Data Analysis. Routledge, 11 New Fetter Lane, London EC4P 4EE 1993, 285 pp, ISBN 0-415-05851-1 (hbk). Dec. 2008

[6.] Gary LD, Bhutta ZA, Cousens S, Taghreed A, Neff Walker, Luc de Bernis, Team ftLNSS (2005). Evidence-based, cost-effective interventions: how many newborn babies can we save? Lancet 2005, 365:977-988

[7.] Ghana Statistical Service (2011). Ghana Multiple Indicator Cluster Survey with an Enhanced Malaria Module and Biomarker, 2011, Final Report. Accra, Ghana.

[8.] Gilani I, Kayani ZA (2014). Household practices of mothers regarding neonatal care in rural \&amp; urban settings of the capital district of Azad Jammu \&amp; Kashmir. Rural Remote Health. 2014;14:2503. Epub 2014 Apr 5. PubMed PMID: 24702463

[9.] Hall, Carl T. (2002). "Study speeds up biological clocks / Fertility rates dip after women hit 27". The San Francisco Chronicle..

[10.] Lawn, J. M. Brian, and R. R. Susan (2001). The Healthy Newborn: A Reference Manual for Program Managers, CARE-CDC Health Initiative Atlanta, 2001.

[11.] Mahama, S. Mariam I. (2014). Patterns and Determinants of Essential Newborn Care Practices in Rural Areas of Northern Ghana. Hindawi Publishing Corporation International Journal of Population Research Volume 2014, Article ID 404387, 10 pages http://dx.doi.org/10.1155/2014/404387

[12.] Mangwi Ayiasi R, Kasasa S, Criel B, Garimoi Orach C, Kolsteren P. (2014). Is antenatal care preparing mothers to care for their newborns? A community-based cross-sectional study among lactating women in Masindi, Uganda. BMC Pregnancy Childbirth. 2014 Mar 25;14:114. doi: 10.1186/1471-2393-14-114. PubMed PMID: 24667001; PubMed Central PMCID: PMC3987096.

[13.] Marah A. (2011). Assessing Household Practices That Influence Neonatal Survival in the AsanteAkim North District of Ashanti Region-Ghana, Kwame Nkrumah University Science and Technology, Kumasi, Ghana, 2011.

[14.] Neupane S, Nwaru BI (2014). Impact of prenatal care utilization on infant care practices in Nepal: a national representative cross-sectional survey. Eur J Pediatr. 2014 Jan;173(1):99-109. doi:

10.1007/s00431-013-2136-y. Epub 2013 Aug 14. PubMed PMID: 23942747

[15.] Under-Five Child Health Policy (2007-2015). Ministry of Health (MOH), Ghana 
South American Journal of Public Health

Special Edition May 2016

\section{Author Note}

Benjamin Aggrey is a District Director of Health Services in the Bawku West District of Ghana. Benjamin Aggrey is now an Integrated PhD in Public Health Student

This research was supported by the staff of Bawku West District Health Directorate, Upper East Region, and Ghana 